\title{
GMR
}

\section{Balance between inflammatory and regulatory cytokines in systemic lupus erythematosus}

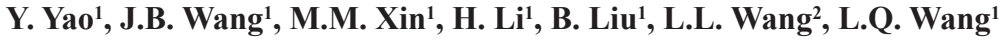 \\ and L. Zhao ${ }^{1}$ \\ ${ }^{1}$ Department of Rheumatology and Clinical Immunology, \\ The Affiliated Hospital of Medical College, Qingdao University, Qingdao, China \\ ${ }^{2}$ Department of Rheumatology and Immunology, Linyi People's Hospital, \\ Linyi, China \\ Corresponding author: J.B. Wang \\ E-mail: summer805_sun@163.com \\ Genet. Mol. Res. 15 (2): gmr. 15027626 \\ Received September 11, 2015 \\ Accepted December 9, 2015 \\ Published May 23, 2016 \\ DOI http://dx.doi.org/10.4238/gmr.15027626
}

\begin{abstract}
To investigate the cytokine profile in serum and cerebrospinal fluid (CSF) from patients with systemic lupus erythematosus (SLE) and central nervous system infection, we measured interferon- $\gamma$ (IFN- $\gamma$ ), interleukin-1 $\beta$ (IL-1 $\beta$ ), IL-4, IL-6, IL-8, IL-10, and IL-17 levels in serum and CSF from 50 SLE patients and 38 matched controls. In patients with active compared to quiescent disease, serum levels were higher for IL-1 $\beta(\mathrm{P}=0.042)$ and IL-17 $(\mathrm{P}=0.041)$ but we found no significant correlation between IL-1 $\beta$ and IL-17 and Systemic Lupus Erythematosus Disease Activity Index (SLEDAI) $(r=$ $0.055, r=0.219$, respectively). IL-10 level in active patients was lower compared to that in quiescent controls $(\mathrm{P}=0.032)$. When comparing specific disease manifestations, IL-1 $\beta$ levels in patients with fever $(\mathrm{P}=$ $0.035)$ and IL-6 $(\mathrm{P}=0.048)$ and IL-8 $(\mathrm{P}=0.048)$ levels in those showing nervous system involvement were higher than in controls. Based on MRI results, we found that only increased cerebral ischemia was associated with increased IFN- $\gamma$ levels $(\mathrm{P}=0.009)$. In neuropsychiatric lupus erythematous patients, CSF levels of IL-6 (P = 0.002), IL-8 (P =
\end{abstract}


0.009), and IL-17 ( $\mathrm{P}=0.034)$ were significantly higher when compared with control patients. IL-10:IL-1 $\beta$ ratio in patients with moderate and quiescent disease was higher than in patients with disease activity $(\mathrm{P}$ $=0.000$ ). Pro-inflammatory adaptive cytokines were elevated during disease flare, while regulatory mediators were elevated during periods of stable disease. Alterations in the balance between inflammatory and regulatory mediators may be targets for novel immunotherapeutic agents for managing autoimmune diseases.

Key words: Systemic lupus erythematosus; Cytokine; Inflammatory; Regulatory

\section{INTRODUCTION}

Systemic lupus erythematosus (SLE) is a chronic, remitting, and relapsing, multisystem autoimmune disease that predominantly affects women. Patients have a broad spectrum of remitting and relapsing clinical symptoms including dermatitis, joint pain, glomerulonephritis and cardiovascular problems (Rahman and Isenberg, 2008). Neurologic and psychiatric manifestations are common in SLE and occur in up to $75 \%$ of patients (Ballok, 2007). Pathophysiologically SLE is characterized by the dysfunction of T, B, and dendritic cells (DC), skewed cytokine production, breakdown of immunological tolerance, and the production of antinuclear autoantibodies (Jenks and Sanz, 2009; Crispín et al., 2010; Perl, 2010; Tsokos, 2011). The disturbance of the cytokine balance is implicated in the pathogenesis of SLE; therefore, cytokine balance might be a candidate target for treating SLE. Recent pharmaceutical advances have led to the possibility of influencing specific cytokines. For example, cytokine-blocking agents have been successful in treating inflammatory joint disease (Maini and Taylor, 2000). However, the usage in SLE has not been as productive probably due to the presence of a more complex clinical and/or immunological profile in SLE, except Belimumab in two large phase III studies demonstrating significant clinical efficacy, although the benefit was not sustained at week 76 (Yildirim-Toruner and Diamond, 2011).

In the present study, we investigated the profile of soluble pro-inflammatory cytokines including IL-1 $\beta$, IL-6, IL-8, IFN- $\gamma$, IL-17, anti-inflammatory cytokine IL-4, and regulatory mediator IL-10 in paired cerebrospinal fluid (CSF) and serum samples from 50 SLE and 38 matched controls. We assessed the correlation between cytokine levels and various clinical manifestations of SLE at different times and determined their potential roles in SLE pathogenesis.

\section{MATIERAL AND METHODS}

\section{Study population}

Whole blood, CSF, and urine samples were collected from SLE patients and matched controls enrolled at the Department of Rheumatology or Neurology of the Affiliation Hospital of QingDao University Medical College. SLE patients met the American College of Rheumatology revised classification criteria for SLE (Hochberg, 1997). All participating subjects gave their written informed consent. The procedures followed were in accordance 
with the ethical standards of the Responsible Committee on Human Experimentation and with the Helsinki Declaration of 1975 (revised in 1983). Evaluation of neuropsychiatric signs and symptoms included neuropsychiatric testing, lumbar puncture, and magnetic resonance imaging (MRI) of the brain. Assays evaluating serum levels of autoantibody and complement $\mathrm{C} 3$ and $\mathrm{C} 4$ were performed at our clinical immunology laboratory. Disease activity was assessed at baseline using the Systemic Lupus Erythematosus Disease Activity Index 2000 update. Active lupus disease was defined as SLEDAI score $>4$ in this study (Gladman et al., 2002). Based on the above criteria, the subjects were divided into four distinct groups: patients with 1) inactive or mild disease $(\mathrm{N}=7), 2)$ disease flare $(\mathrm{N}=43), 3)$ central nervous system infection $(\mathrm{N}=19)$, or 4) brain trauma $(\mathrm{N}=19)$.

\section{Immunoassay of cytokines}

Ten-milliliter samples of peripheral blood and CSF collected from patients and controls were centrifuged at $2000 \mathrm{~g}$. The supernatant was immediately $(<30 \mathrm{~min})$ collected and stored at $-80^{\circ} \mathrm{C}$ for cytokine measurement. Serum samples were collected before an increase in dose of corticosteroid or immunosuppressive drugs for patients with active disease. Cytokines were measured using ELISA kits from eBioscience (San Diego, CA, USA). The detection limits for IFN- $\gamma$, IL-1 $\beta$, IL-4, IL-6, IL-8, IL-10, and IL-17 are 3, 4, 2, 2, 2, 15, and $10 \mathrm{pg} / \mathrm{mL}$, respectively.

\section{Statistical analysis}

Data analysis was performed by SPSS 20.0. All data are reported as means \pm standard deviation or median for the various cytokines as their distribution was highly skewed. Analysis of variance (ANOVA) was used to compare continuous variables between groups. A simple linear regression analysis was used to evaluate the correlation between the two parameters. $\mathrm{P}$ $<0.05$ was considered statistically significant. Spearman's correlation was used for correlation analysis. $|r| \geq 0.70$ indicates substantial agreement, $0.40 \leq|r| \leq 0.70$ indicates moderate agreement, $|\mathrm{r}| \leq 0.40=$ slight agreement.

\section{RESULTS}

\section{Characteristics of the study population}

50 patients with SLE ( 45 females, 5 males) and 38 controls ( 30 females, 8 males) were recruited. The mean \pm SD age of these patients was $37.5 \pm 9.1$ years with a disease duration of $7.2 \pm 5.8$ years. Thirty-six patients had active disease with a SLEDAI of $12.0 \pm 4.9$ (range 2-21).

\section{Cytokine levels in relation to clinical profiles}

IL-1 $\beta$ and IL-17 levels in patients with disease activity were higher than in patients with moderate and quiescent disease $(\mathrm{P}=0.042, \mathrm{P}=0.041$, respectively $)$ but we found no significant correlation between IL-1 $\beta$ and IL-17 and disease activity $(\mathrm{r}=0.055, \mathrm{P}=0.705$; $\mathrm{r}=0.219, \mathrm{P}=0.126$, respectively). Interestingly, active patients had lower levels of IL-10 compared to healthy controls $(\mathrm{P}=0.032)$. Serum levels of other cytokines were not different between these groups of patients (Table 1). When analyzed by the presence of specific disease 
manifestations, we found a higher IL-1 $\beta$ level in fever patients $(P=0.035)$ and IL-6 and IL-8 levels in nervous system involvement $(\mathrm{P}=0.048, \mathrm{P}=0.048$, respectively). Among standard laboratory findings, i.e. WBC, CRP, complement, anti-ds-DNA antibody, and there was no significant association between these cytokines and laboratory findings among all SLE patients (data not shown). Furthermore, we had also evaluated the associations between these cytokines and all MRI outcomes, revealing that cerebral ischemia was significant associated with increased IFN- $\gamma$ levels $(\mathrm{P}=0.009)$.

Table 1. Cytokine levels in serum and CSF from SLE patients and controls (means \pm SD).

\begin{tabular}{l|c|c|c|c|c}
\hline Cytokines & Brain trauma & \multirow{2}{*}{ Mild disease } & \multicolumn{2}{|c|}{ Disease flare } & Central nervous system infection \\
\cline { 3 - 5 } & & & No-NPSLE & NPSLE & \\
\hline IFN- $\gamma(\mathrm{S})$ & $21.92 \pm 8.20$ & $19.04 \pm 7.91$ & $19.63 \pm 6.91$ & $18.41 \pm 8.44$ & $21.92 \pm 8.20$ \\
\hline IFN- $\gamma(\mathrm{C})$ & $19.56 \pm 6.63$ & $15.99 \pm 8.79$ & $16.87 \pm 7.05$ & $19.77 \pm 9.82$ & $19.56 \pm 6.63$ \\
\hline IL-1 $\beta(\mathrm{S})$ & $15.91 \pm 5.86$ & $13.59 \pm 5.33$ & $19.87 \pm 7.48$ & $19.69 \pm 7.58$ & $15.91 \pm 5.86$ \\
\hline IL-1 $\beta(\mathrm{C})$ & $16.34 \pm 3.73$ & $10.70 \pm 1.95$ & $16.60 \pm 8.61$ & $15.24 \pm 6.52$ & $16.34 \pm 3.73$ \\
\hline IL-4(S) & $7.66 \pm 3.10$ & $7.74 \pm 2.69$ & $8.41 \pm 3.49$ & $7.45 \pm 3.09$ & $7.66 \pm 3.10$ \\
\hline IL-4(C) & $10.65 \pm 3.41$ & $10.93 \pm 3.32$ & $10.05 \pm 3.60$ & $10.13 \pm 3.31$ & $10.65 \pm 3.41$ \\
\hline IL-6(S) & $76.00 \pm 14.74$ & $20.89 \pm 8.28$ & $17.29 \pm 7.26$ & $24.29 \pm 10.98^{*}$ & $76.00 \pm 14.74$ \\
\hline IL-6(C) & $133.77 \pm 26.50$ & $37.53 \pm 39.10$ & $49.60 \pm 59.27$ & $83.52 \pm 31.10^{*}$ & $133.77 \pm 26.50^{*}$ \\
\hline IL-8(C) & $219.65 \pm 67.44$ & $132.33 \pm 50.67$ & $126.25 \pm 77.64$ & $165.81 \pm 61.18^{*}$ & $219.65 \pm 67.44$ \\
\hline IL-10(S) & $410.37 \pm 154.51$ & $81.71 \pm 34.90$ & $192.38 \pm 175.89$ & $265.57 \pm 137.99^{*}$ & $410.37 \pm 154.51^{*}$ \\
\hline IL-10(C) & $8.75 \pm 4.44$ & $8.84 \pm 2.76$ & $6.94 \pm 1.50$ & $7.00 \pm 2.14$ & $8.75 \pm 4.44$ \\
\hline IL-17(S) & $23.46 \pm 5.85$ & $6.19 \pm 2.17$ & $10.04 \pm 6.84$ & $7.68 \pm 3.27$ & $8.46 \pm 5.85$ \\
\hline IL-17(C) & $29.11 \pm 11.31$ & $16.10 \pm 2.98$ & $15.98 \pm 9.10$ & $21.03 \pm 8.30^{*}$ & $23.74 \pm 9.50$ \\
\hline
\end{tabular}

$\mathrm{S}=$ serum; $\mathrm{C}=\mathrm{CSF} .{ }^{*}$ Significantly different $(\mathrm{P}<0.05)$.

\section{Intrathecal cytokine profiling among patients}

IL-6, IL-8, and IL-17 levels were higher in NPLE patients compared with SLE (P = $0.002, \mathrm{P}=0.009, \mathrm{P}=0.034$, respectively). IL-6, IL-8, and IL-17 were higher in CNS infection patients than in NPLE patients ( $\mathrm{P}=0.000, \mathrm{P}=0.001, \mathrm{P}=0.006$, respectively).

\section{IL-10:IL-1 $\beta$ ratio in serum and CSF}

The IL-10:IL-1 $\beta$ ratio in serum among patients with moderate and quiescent disease was higher than in patients with disease activity $(\mathrm{P}=0.000)$. There was no difference in CSF (data not shown).

\section{DISCUSSION}

Cytokines are important mediators of intercellular communication and orchestrate the interaction of immune cells during immune responses, for instance, interleukins (ILs), interferons (IFNs), and growth factors. Lower cytokine production contributes to immune dysfunction and causes tissue inflammation and organ damage. Cytokines are important in the pathogenesis of SLE. Indeed, a different quantitative and/or qualitative expression pattern of cytokines might determine the activation and suppression of signals in SLE.

In this study on SLE in an continuous patient group, we found a heterogeneous cytokine profile in both serum and CSF and no correlation between disease manifestations or severity and cytokine level except for IL-1 $\beta$ in serum. IL-1 is considered a "master" cytokine 
in the pro-inflammatory cytokine network (Voronov et al., 2006) and consists of two agonistic proteins (IL- $1 \alpha$ and IL-1 $\beta$ ) and the inhibitor IL-1 receptor antagonist (IL-1Ra). While IL-1 $\beta$ is the secreted active form produced mainly by macrophages, few studies have investigated IL$1 \beta$ in SLE (Dean et al., 2000; Aringer and Smolen, 2004). We found IL-1 $\beta$ is related to fever. Fever is defined as a regulated increase in internal body temperature to levels above normal. Dinarello (1984) found IL-1 was the same as the fever-inducing factor produced by leukocytes and known as leukocyte pyrogen. He also outlined in a review article the mechanism by which leukocyte pyrogen was likely to initiate fever and predicted the likely mechanism whereby antipyretics prevent the synthesis of COX metabolites and therefore reduce fever (Dinarello and Wolff, 1982). These proposed mechanisms still hold true today (Steinman L, 2008). Bartfai and his teams have refined that IL- $1 \beta$-mediated induction of sphingomyelinase via the receptor for IL-1 mediates a rapid febrile response that circumvents the need for PGE2, which requires NF- $\mathrm{kB}$ induction of transcription of $\mathrm{COX} 2$, a process that takes around 30 mins (Sanchez-Alavez et al., 2006). We found an elevated IL-1b level in fever patients, which may be consistent with this study. Previous clinical studies have suggested that serum IFN-g and IL-17 levels are correlated with lupus nephritis and SLEDAI, respectively (Doreau et al., 2009). However, other research suggests that serum IL-17 is not related to the levels of proteinuria or parameters of disease activity, including SLEDAI, serum levels of anti-dsDNA antibody, or C3 and C4. This suggests that elevated IL-17 is involved in the etiology of SLE but is not solely related to renal involvement in SLE patients (Zhao et al., 2010). We found that SLE patients with inactive or mild disease did not have differences in IFN-r, IL-4, and IL-10 levels compared to healthy controls, consistent with other studies (Amit M et al., 2000; Gröndal et al., 2000; Enghard et al., 2006; Kozora et al., 2012; Ballantine et al., 2014). Some clinical studies are contradictory to these results. In an open-label pilot study a single dose of murine anti-IL-10 mAb (B-N10) was given to six patients with active steroid-dependent SLE and led to declining mean daily prednisolone dose (Llorente et al., 2000), however there are fundamental differences between human and murine SLE (Ballok, 2007). No studies have evaluated humanized anti-IL-10 mAb thus far (Yildirim-Toruner and Diamond, 2011).

Immune cells exert many of their effector and immunoregulatory functions through cytokine release. Most cytokines investigated are dysregulated in SLE patients, suggesting that variability of inflammatory mediators is important in active SLE patients (Tokano et al., 1999; Gómez et al., 2004; Mok et al., 2010). In this study, SLE patients have significantly higher IL$1 \beta$ and lower IL-10 levels during an early flare compared to non-flare SLE patients, indicating a disrupted balance between inflammatory and regulatory mediators. The IL-10:IL-1 $\beta$ ratio implicates an increased regulatory anti-inflammatory state in stable periods of SLE. Compared to matched patients with stable disease, the decrease in anti-inflammatory and immunoregulatory cytokine IL-10, which is required for T-regulatory cell development and propagation, may corroborate a failure of active regulation in the early disease flare. In addition, in contrast to pro-inflammatory mediators, regulatory cytokines IL-10 levels are higher in stable SLE patients, suggesting context-dependent regulatory roles for these cytokines (Munroe et al., 2014). IL-10 is expressed by cells of the adaptive immune system including Th1, Th2, Th17, and Treg cells, and cells of the innate immune system (DCs, macrophages, NK, eosinophils, and neutrophils) (Saraiva and O'Garra, 2010; Carter et al., 2011). IL-10 is a key cytokine regulating immune responses, and the absence results in spontaneous inflammatory disorders and exacerbated inflammation (Li and Flavell, 2008; Ouyang et al., 2011). In this study, we did not measure serum TGF- $\beta$, the levels of which diminish in association with disease activity, 
lymphocyte subsets, and damage in SLE patients (Becker-Merok et al., 2010). Therefore, we hypothesize that for treating SLE, we should focus on therapeutic agents that regulate the immune balance between inflammatory and regulatory mediators rather than on those that exclusively target pro-inflammatory cytokines.

Our results show that despite the diversity and heterogeneous nature of the NP manifestations of SLE, the levels of several molecules, including those of IL-6, IL-8, IL17 , are increased in the CSF of most patients during NP activity. Recent studies show that the presence of intrathecal IL-6 and IL-8 leads to the synthesis of matrix metalloproteinase 9 (MMP-9), which results in brain parenchyma damage and the release of neuronal and astrocytic degradation products that terminate in MRI-verifiable lesions and clinical states of brain deficiency (Trysberg et al., 2004). Thus far, little information is known about the levels of intrathecal IL-17. Unlike in multiple sclerosis, IL-17 is not crucial in the pathogenesis of NPLE (Garrett-Sinha et al., 2008; Miossec et al., 2009). Our data indicate that increased IFN- $\gamma$ levels are associated with cerebral ischemia by MRI. Vascular compromise resulting in brain ischemia is frequently related to antiphospholipid (APL) antibodies. The expression of intrathecal IL-6 and chemokines during the clinical activity of NPLE is different than the expression in the peripheral blood (Mok and Lau, 2003; Lit et al., 2006), suggesting that these molecules are produced in situ and that the damage seen in NPLE does not require factors derived from blood. These results indicate that neurons, microglia, and astroglia can all serve as both targets and sources of chemokines (Giulian et al., 1993), which represents an innate immune response.

We acknowledge some limitations of our study. The cytokine panel we studied represents a significant portion of the inflammatory process in SLE, but there are limitations imposed by our selection of cytokines. In addition, our study was also limited by small sample size, cross-sectional design, and multiple statistical comparisons. Serum levels of cytokines may not reflect actual inflammation as their functional state and it is an oversimplification to assume that an individual cytokine is pro- or anti-inflammatory. The precise proportions of cytokine levels in serum and brain are likely to be more important than absolute levels of a single cytokine. Studies of a larger group of patients with active and inactive disease are needed to better evaluate the kinetics of these cytokines and their relationship with disease activity and clinical response to treatment in SLE patients. Although blood and CSF samples were collected from active patients prior to augmentation of corticosteroids and immunosuppressive drugs, we cannot exclude a possible influence of concurrent medications on cytokine levels.

Cytokine-modulating therapies are not currently in clinical use for the treatment of SLE. Novel therapies restoring the immune balance between inflammatory and regulatory mediators are essential for future therapeutic strategies and to ensure appropriate treatment to avoid severe complications.

\section{Conflicts of interest}

The authors declare no conflict of interest.

\section{REFERENCES}

Amit M, Mor A, Weissgarten J, Rosenberg R, et al. (2000). Inactive systemic lupus erythematosus id associated with a normal stimulated $\mathrm{Th}(1) / \mathrm{Th}(2)$ cytokine secretory pattern. Cytokine 12: 1405-1408. http://dx.doi.org/10.1006/ cyto.2000.0724 
AringerMandSmolenJS(2004). Tumournecrosisfactorandotherproinflammatorycytokinesinsystemiclupuserythematosus: a rationale for therapeutic intervention. Lupus 13: 344-347.http://dx.doi.org/10.1191/09612033031u1024oa

Ballantine LE, Ong J, Midgley A, Watson L, et al. (2014). The pro-inflammatory potential of T cells in juvenile-onset systemic lupus erythematosus. Pediatr. Rheumatol. Online J. 12: 4.

Ballok DA (2007). Neuroimmunopathology in a murine model of neuropsychiatric lupus. Brain Res. Brain Res. Rev. 54: 67-79. http://dx.doi.org/10.1016/j.brainresrev.2006.12.003

Becker-Merok A, Eilertsen GØ and Nossent JC (2010). Levels of transforming growth factor-beta are low in systemic lupus erythematosus patients with active disease. J. Rheumatol. 37: 2039-2045. http://dx.doi.org/10.3899/jrheum.100180

Carter NA, Vasconcellos R, Rosser EC, Tulone C, et al. (2011). Mice lacking endogenous IL-10-producing regulatory B cells develop exacerbated disease and present with an increased frequency of Th1/Th17 but a decrease in regulatory T cells. J. Immunol. 186: 5569-5579. http://dx.doi.org/10.4049/jimmunol.1100284

Crispín JC, Kyttaris VC, Terhorst C and Tsokos GC (2010). T cells as therapeutic targets in SLE. Nat. Rev. Rheumatol. 6: 317-325.http://dx.doi.org/10.1038/nrrheum.2010.60

Dean GS, Tyrrell-Price J, Crawley E and Isenberg DA (2000). Cytokines and systemic lupus erythematosus. Ann. Rheum. Dis. 59: 243-251.http://dx.doi.org/10.1136/ard.59.4.243

Dinarello CA (1984). Interleukin-1. Rev. Infect. Dis. 6: 51-95. http://dx.doi.org/10.1093/clinids/6.1.51

Dinarello CA and Wolff SM (1982). Molecular basis of fever in humans. Am. J. Med. 72: 799-819. http://dx.doi. org/10.1016/0002-9343(82)90548-4

Doreau A, Belot A, Bastid J, Riche B, et al. (2009). Interleukin 17 acts in synergy with B cell-activating factor to influence B cell biology and the pathophysiology of systemic lupus erythematosus. Nat. Immunol. 10: 778-785. http://dx.doi. org/10.1038/ni.1741

Enghard P, Langnickel D and Riemekasten G (2006). T cell cytokine imbalance towards production of IFN-g and IL-10 in NZB/W F1 lupus-prone mice is associated with autoantibody levels and nephritis. Scand. J. Rheumatol. 35: 209-216. http://dx.doi.org/10.1080/03009740500417791

Garrett-Sinha LA, John S and Gaffen SL (2008). IL-17 and the Th17 lineage in systemic lupus erythematosus. Curr. Opin. Rheumatol. 20: 519-525. http://dx.doi.org/10.1097/BOR.0b013e328304b6b5

Giulian D, Vaca K and Corpuz M (1993). Brain glia release factors with opposing actions upon neuronal survival. $J$. Neurosci. 13: 29-37.

Gladman DD, Ibañez D and Urowitz MB (2002). Systemic lupus erythematosus disease activity index 2000. J. Rheumatol. 29: 288-291.

Gómez D, Correa PA, Gómez LM, Cadena J, et al. (2004). Th1/Th2 cytokines in patients with systemic lupus erythematosus: is tumor necrosis factor alpha protective? Semin. Arthritis Rheum. 33: 404-413. http://dx.doi. org/10.1016/j.semarthrit.2003.11.002

Gröndal G, Gunnarsson I, Rönnelid J, Rogberg S, et al. (2000). Cytokine production, serum levels and disease activity in systemic lupus erythematosus. Clin. Exp. Rheumatol. 18: 565-570.

Hochberg MC (1997). Updating the American College of Rheumatology revised criteria for the classification of systemic lupus erythematosus. Arthritis Rheum. 40: 1725-1734.http://dx.doi.org/10.1002/art.1780400928

Jenks SA and Sanz I (2009). Altered B cell receptor signaling in human systemic lupus erythematosus. Autoimmun. Rev. 8: 209-213.http://dx.doi.org/10.1016/j.autrev.2008.07.047

Kozora E, Filley CM, Zhang L, Brown MS, et al. (2012). Immune function and brain abnormalities in patients with systemic lupus erythematosus without overt neuropsychiatric manifestations. Lupus 21: 402-411. http://dx.doi. org/10.1177/0961203311429116

Li MO and Flavell RA (2008). Contextual regulation of inflammation: a duet by transforming growth factor-b and interleukin-10. Immunity 28: 468-476.http://dx.doi.org/10.1016/j.immuni.2008.03.003

Lit LC, Wong CK, Tam LS, Li EK, et al. (2006). Raised plasma concentration and ex vivo production of inflammatory chemokines in patients with systemic lupus erythematosus. Ann. Rheum. Dis. 65: 209-215.http://dx.doi.org/10.1136/ ard.2005.038315

Llorente L, Richaud-Patin Y, García-Padilla C, Claret E, et al. (2000). Clinical and biologic effects of anti-interleukin-10 monoclonal antibody administration in systemic lupus erythematosus. Arthritis Rheum. 43: 1790-1800.http://dx.doi. org/10.1002/1529-0131(200008)43:8<1790::AID-ANR15>3.0.CO;2-2

Maini RN and Taylor PC (2000). Anti-cytokine therapy for rheumatoid arthritis. Annu. Rev. Med. 51: 207-229. http:// dx.doi.org/10.1146/annurev.med.51.1.207

Miossec P, Korn T and Kuchroo VK (2009). Interleukin-17 and type 17 helper T cells. N. Engl. J. Med. 361: 888-898. http://dx.doi.org/10.1056/NEJMra0707449

Mok CC and Lau CS (2003). Pathogenesis of systemic lupus erythematosus. J. Clin. Pathol. 56: 481-490. http://dx.doi. org/10.1136/jep.56.7.481 
Mok MY, Wu HJ, Lo Y and Lau CS (2010). The relation of interleukin 17 (IL-17) and IL-23 to Th1/Th2 cytokines and disease activity in systemic lupus erythematosus. J. Rheumatol. 37: 2046-2052. http://dx.doi.org/10.3899/ jrheum. 100293

Munroe ME, Vista ES, Guthridge JM, Thompson LF, et al. (2014). Proinflammatory adaptive cytokine and shed tumor necrosis factor receptor levels are elevated preceding systemic lupus erythematosus disease flare. Arthritis Rheumatol. 66: 1888-1899. http://dx.doi.org/10.1002/art.38573

Ouyang W, Rutz S, Crellin NK, Valdez PA, et al. (2011). Regulation and functions of the IL-10 family of cytokines in inflammation and disease. Annu. Rev. Immunol. 29: 71-109. http://dx.doi.org/10.1146/annurevimmunol-031210-101312

Perl A (2010). Pathogenic mechanisms in systemic lupus erythematosus. Autoimmunity 43: 1-6. http://dx.doi. org $/ 10.3109 / 08916930903374741$

Rahman A and Isenberg DA (2008). Systemic lupus erythematosus. N. Engl. J. Med. 358: 929-939. http://dx.doi. org/10.1056/NEJMra071297

Sanchez-Alavez M, Tabarean IV, Behrens MM and Bartfai T (2006). Ceramide mediates the rapid phase of febrile response to IL-1beta. Proc. Natl. Acad. Sci. USA 103: 2904-2908. http://dx.doi.org/10.1073/pnas.0510960103

Saraiva M and O'Garra A (2010). The regulation of IL-10 production by immune cells. Nat. Rev. Immunol. 10: 170-181. http://dx.doi.org/10.1038/nri2711

Steinman L (2008). Nuanced roles of cytokines in three major human brain disorders. J. Clin. Invest. 118: 3557-3563. http://dx.doi.org/10.1172/JCI36532

Tokano Y, Morimoto S, Kaneko H, Amano H, et al. (1999). Levels of IL-12 in the sera of patients with systemic lupus erythematosus (SLE)--relation to Th1- and Th2-derived cytokines. Clin. Exp. Immunol. 116: 169-173. http://dx.doi. org/10.1046/j.1365-2249.1999.00862.x

Trysberg E, Blennow K, Zachrisson O and Tarkowski A (2004). Intrathecal levels of matrix metalloproteinases in systemic lupus erythematosus with central nervous system engagement. Arthritis Res. Ther. 6: R551-R556. http:// dx.doi.org/10.1186/ar1228

Tsokos GC (2011). Systemic lupus erythematosus. N. Engl. J. Med. 365: 2110-2121. http://dx.doi.org/10.1056/ NEJMra1100359

Voronov E, Dayan M, Zinger H, Gayvoronsky L, et al. (2006). IL-1 beta-deficient mice are resistant to induction of experimental SLE. Eur. Cytokine Netw. 17: 109-116.

Yildirim-Toruner C and Diamond B (2011). Current and novel therapeutics in the treatment of systemic lupus erythematosus. J. Allergy Clin. Immunol. 127: 303-312, quiz 313-314.http://dx.doi.org/10.1016/j.jaci.2010.12.1087

Zhao XF, Pan HF, Yuan H, Zhang WH, et al. (2010). Increased serum interleukin 17 in patients with systemic lupus erythematosus. Mol. Biol. Rep. 37: 81-85. http://dx.doi.org/10.1007/s11033-009-9533-3 